\title{
Glans metastatic extra-nodal natural killer/T-cell lymphoma, nasal-type with HDAC inhibitor as maintenance therapy: a rare case report with literature review
}

\author{
Chunxi Fu ${ }^{1}$, Chunli Yang ${ }^{1}$, Qiaoer Li $^{1}$, Ling Wang ${ }^{2}$, Liqun Zou ${ }^{1}$ \\ ${ }^{1}$ Department of Oncology, ${ }^{2}$ Department of Dermatology, West China Hospital, Sichuan University, Chengdu, China \\ Correspondence to: Liqun Zou. Department of Oncology, West China Hospital, Sichuan University, No. 37, Guo Xue Xiang, Chengdu 610041, China. \\ Email: hxlcyxy@163.com.
}

\begin{abstract}
Extra-nodal natural killer/T-cell lymphoma (ENKTL) is an aggressive lymphoid malignancy and advanced ENKTL is characterized by a dismal survival outcome. Distant metastasis is frequently common in advanced ENKTLs, while the glans is rarely involved. Here we report a 67-year-old Chinese male with glans metastasis ENKTL, nasal type secondary from the nasal cavity, treated with modified SMILE regimen plus glans radiotherapy and achieved a complete remission (CR). Since the poor prognosis of metastatic ENKTL and unfitness for hematological stem cell transplant (HSCT), we administered histone deacetylase (HDAC) inhibitor Chidamide as maintenance therapy, which was resulted in a progression-free survival (PFS) of 12 months. To investigate the gene alternations, the whole exon sequencing and the 446-gene panel sequencing were performed and we found the BCOR, KRAS, CDKN1B, XPO1, DOT1L genes mutations. Best to our knowledge, this is a glans metastasis ENKTL with the longest PFS and the most successful treatment.
\end{abstract}

Keywords: Extra-nodal natural killer/T-cell lymphoma (ENKTL); glans; histone deacetylase inhibitors (HDAC inhibitors); gene sequencing; case report

Submitted Oct 29, 2019. Accepted for publication Apr 10, 2020.

doi: 10.21037/apm-19-430b

View this article at: http://dx.doi.org/10.21037/apm-19-430b

\section{Introduction}

Extra-nodal natural killer/T-cell lymphoma (ENKTL) is a common extra-nodal aggressive lymphoma with higher incidence rate in Asia, Latin America and South America than Western countries, accounted for $1-10 \%$ of NonHodgkin's lymphoma in comparison with $12.0-17.1 \%$ in China $(1,2)$. ENKTL, nasal type often primarily occurs in the midline facial structure, including the nasal cavity, nasopharynx (sinus), oropharynx and throat, and the most frequently affected or metastatic sites outside the nose including the skin and gastrointestinal tract. Extension to the male genitourinary system such as penis or glans is very rare. L-asparaginase-based chemotherapy is a preferred regimen for early and advanced ENKTL, however, there is still $17.8 \%$ patients progress to recurrence or metastasis (3). The frequent metastatic sites in ENKTL is including the skin and gastrointestinal tract, extending to the male genitourinary system such as penis is very rare. In literature, there was only three secondary penile ENKTL English cases and one primary ENKTL Chinese case reported, in which the longest survival time was only 4 months after second recurrence (4-7). There is no report about maintenance therapy for peripheral T-cell lymphoma (PTCL) with histone deacetylase (HDAC) inhibitors, let alone ENKTLs. Here, we reported a case of penile metastasis ENKTL secondary from the nasal cavity reached a complete remission (CR) after treatment and the PFS time is 12 months undergoing with HDAC inhibitor as maintenance therapy. We present the following case in accordance with the CARE Reporting Checklist.

\section{Case presentation}

A 67-year-old Chinese male presented to our hospital in 

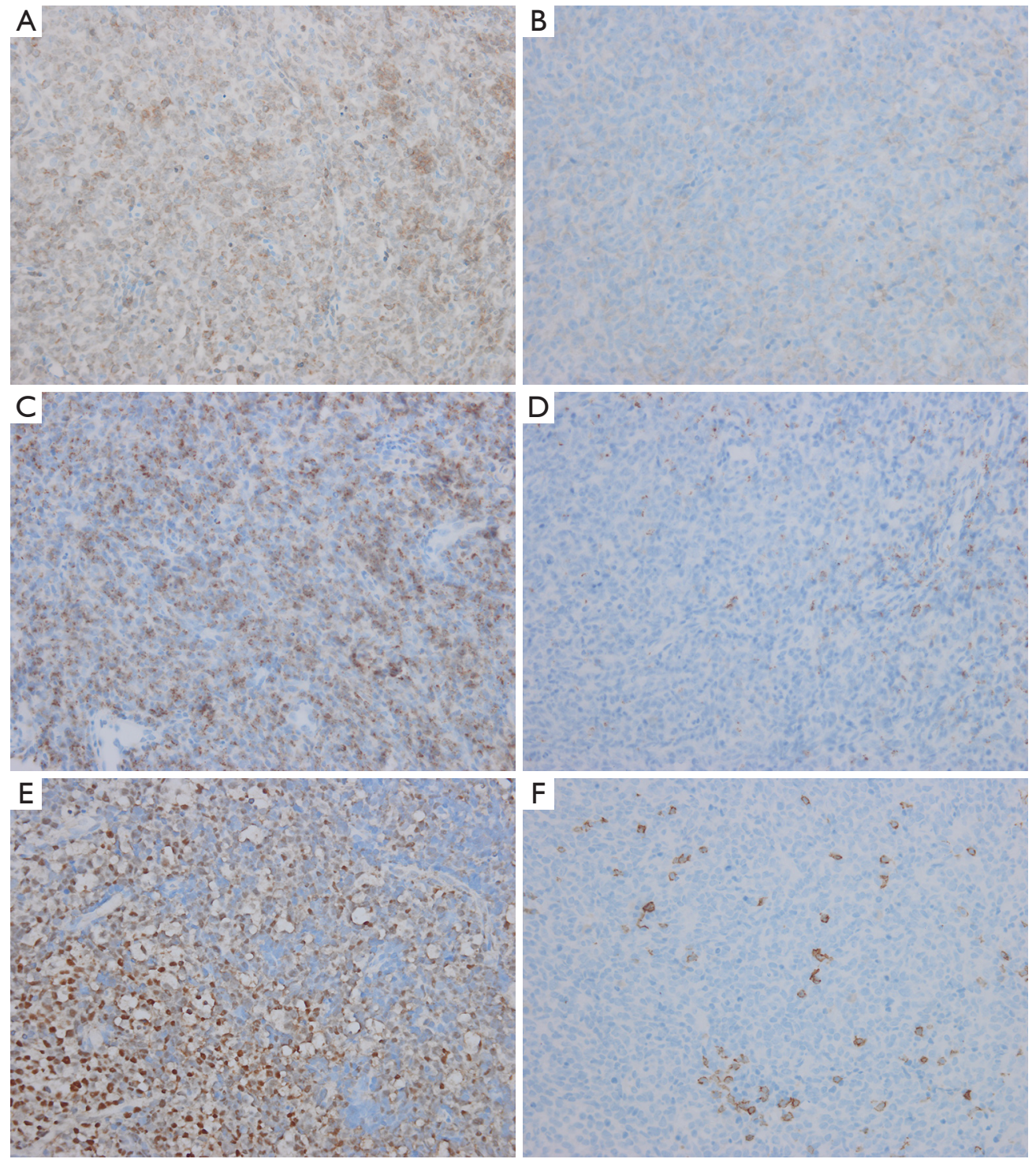

Figure 1 Microscopic features of tumor cells from the left nasal cavity. (A,B,C,D) Immunohistochemical staining showed positive reactivity for CD3\& (×200), CD56 (×200), TIA-1 (×200), granzyme B (×200), respectively; (E) in situ hybridization for EBV-encoded RNA showed positive reaction in atypical cells $(\times 200)$; (F) immunohistochemical staining showed negative CD20 $(\times 200)$. EBV, Epstein-Barr virus.

January 2017, complaining nasal intermittently congesting and bleeding for 3 months, without fever, night sweat, weight loss and other symptoms. The complete blood count (CBC), lactate dehydrogenase (LDH) was normal and Epstein-Barr virus-DNA (EBV-DNA) copy numbers were negative. Nasopharyngeal fiberoscopy detected a mass with part necrosis in the left nasal cavity, which was diagnosed as ENKTL, nasal type with EBV-encoded small RNAs 1/2 (EBER 1/2) positive by biopsy (Figure 1). In addition, the ${ }^{18} \mathrm{~F}-\mathrm{FDG}$ PET/CT scan showed no lymphoma invasion except abnormal increase in glucose metabolism in the left nasal cavity (Deauville score 5). P-GemOx scheme (gemcitabine, oxaliplatin and pegaspargase) chemotherapy was applied for four cycles and followed by local nasal radiotherapy by ${ }^{60} \mathrm{Co}$ (200 cGy, 25 times; total, 5,000 cGy), leading to an unconfirmed complete remission $(\mathrm{CRu})$ by contrasted nuclear magnetic resonance imaging (MRI) for nasopharyngeal.

Nonetheless, 8 months later after radiotherapy, the patient appeared balanitis without response to anti-infection 

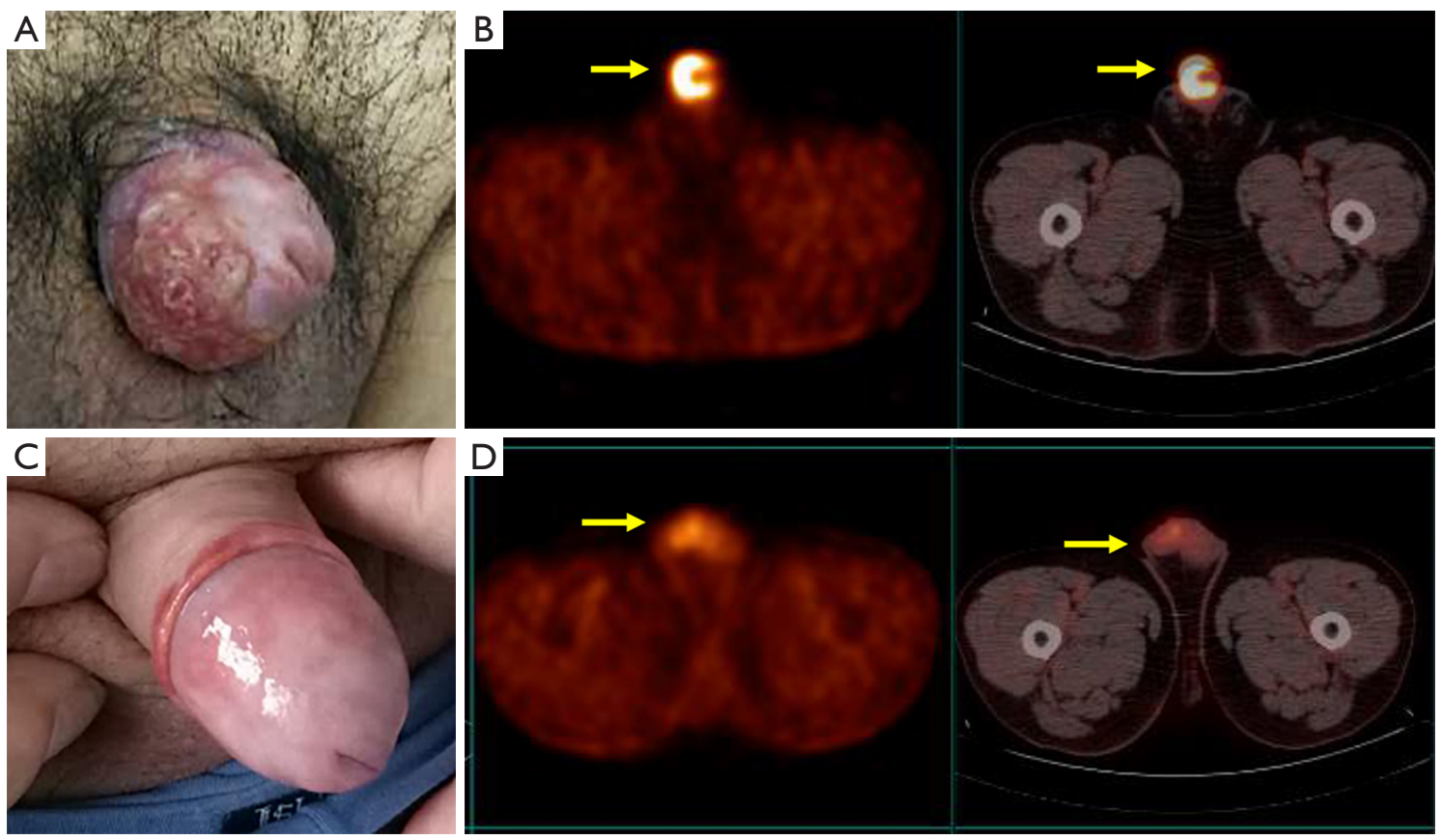

Figure 2 Clinical manifestation and PET/CT imaging of the glans. (A) showed the lesion on the glans; (B) showed the metastasize of the glans in PET/CT image; (C) depicts the CR of the disease in the same area after treatment; (D) is the PET/CT image when the patient achieved CR. The position indicated by the arrow represents the metabolic changes of the focus in PET/CT. CR, complete remission.

treatment (Figure 2A). The physical examination found a $3 \mathrm{~cm} \times 3 \mathrm{~cm}$ mass in the glans penis covered with ulcers. In workup, white blood cell and neutrocyte proportion was normal, and human immunodeficiency virus (HIV) was negative, while EBV-DNA copies were $2.79 \times 10 \mathrm{E} 01$ copies/ $\mathrm{mL}$, which was higher than before, indicating a possibility of tumor relapsed. The ${ }^{18} \mathrm{~F}$-FDG PET/CT scan revealed a higher SUV value in the penis than liver and no other positive findings (Figure $2 \mathrm{~B}$ ). Cerebrospinal fluid biopsy was also examined to be negative. Accordingly, we operated biopsy of the glans ulcer and confirmed the ENKTL diagnosis. We administered modified-SMILE regimen (methotrexate, dexamethasone, ifosfamide, etoposide and L-asparaginase) with intrathecal chemotherapy (cytarabine, methotrexate and dexamethasone) for four cycles. The glans ulceration was rapidly healing following the first cycle and a CR was attained after two cycles confirmed by ${ }^{18} \mathrm{~F}-\mathrm{FDG}$ PET-CT scan (Figure 2C,D). Immunohistochemical staining showed the tissue was positive for CD3, TIA-1, and EBER, negative for CD56, D20. There was no doubt of the diagnosis of ENKTL with metastasis of penis (Figure 3). Since the poor prognosis of metastatic ENKTL and unfitness for hematological stem cell transplant (HSCT), we applied glans radiotherapy (200 cGy/23 f/4,600 cGy) and following with HDAC inhibitor Chidamide (epidaza, $20 \mathrm{mg}$, biw, po) as maintenance therapy, which was resulted in a progressionfree survival (PFS) of 12 months (the timeline see Figure 4). Of note, the Chidamide is less toxic and no grade 3-4 adverse events were found in the course of medication.

To investigate the genetic aberration in this case, we performed whole exome sequencing and targeted 446-gene panel deep sequencing of the patient's tumor tissues and peripheral blood samples (Table 1), found BCOR, KRAS, CDKN1B, XPO1 gene mutations.

\section{Discussion}

Penis is a rare site involved in malignant tumors and the reported incidence ranging from 0.58 to 1.3 cases $/ 100,000$ individuals, in which squamous cell carcinoma is the most common type $(8,9)$. We searched literatures in PubMed and found only four reported penis infiltrated ENKTL metastasis cases (Table 2) available. These cases predispose to middle age and the clinical presentations of which are all manifested as nodules, masses and ulceration. The longest survival time was only 4 months after second recurrence despite the use of L-asparaginase-based chemotherapy. Li et al. summarized the clinical characteristics of penile 

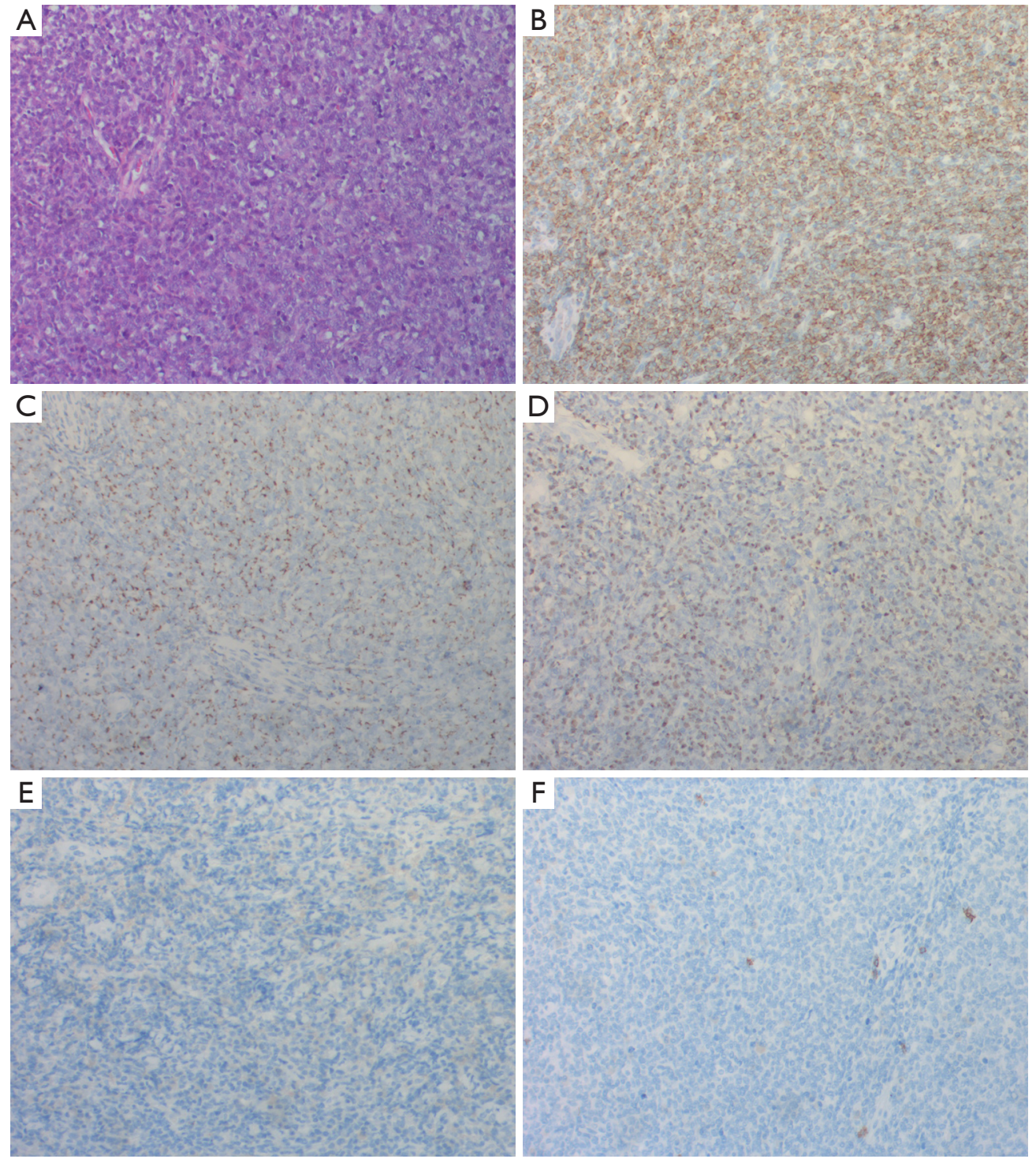

Figure 3 Microscopic features of mass of glans. (A) Histological examinations stained with H\&E revealed infiltration of medium-sized cells with irregular nuclei, inconspicuous nucleoli, and many apoptotic bodies (H\&E stains $\times 200)$; $(\mathrm{B}, \mathrm{C})$ immunohistochemical staining showed positive reactivity for CD3 $(\times 200)$, TIA-1 $(\times 200)$, respectively; (D) in situ hybridization for EBV-encoded RNA showed positive reaction in atypical cells (×200); (E,F) immunohistochemical staining showed negative CD56 and CD20 (×200). H\&E, hematoxylin and eosin; EBV, Epstein-Barr virus.

lymphoma and found that the clinical presentations of penile lymphomas are often non-specific and subtle, including nodules, masses, ulceration and diffuse penile swelling, and more rare symptoms include dysuria and phimosis (6). Among the reviewed cases, 69\% exhibited ulceration, which may be a sign that indicates a possible diagnosis of penile lymphoma. As mentioned above, it is highlight re-biopsy for any suspicious site to make a definite diagnosis, especially for patients with a history of cancer.

Penile metastatic lymphoma appears to indicate a worse prognosis, and there has no standard treatment for relapsed or refractory ENKTLs (R/R ENKTLs), much less penile metastasis. Clinical studies have suggested that L-asparaginase-based chemotherapy is preferred for advanced ENKTL, and SMILE regimen is effective in both initial stage III-IV and R/R NKTCL, with objective 


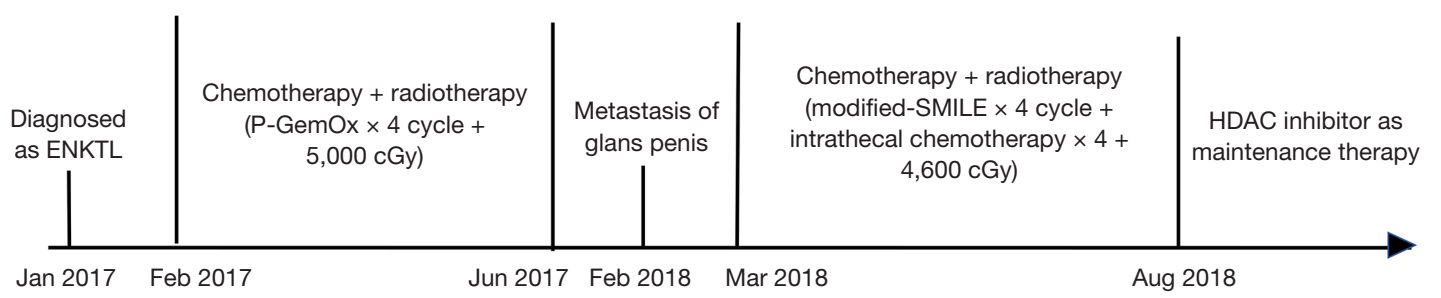

Figure 4 Timeline. The information of the patient with glans metastatic ENKTL, nasal-type. ENKTL, extra-nodal natural killer/T-cell lymphoma; HDAC, histone deacetylase.

Table 1 Gene mutations identified by next-generation sequencing

\begin{tabular}{lccc}
\hline Gene & Mutation & AF in peripheral blood, \% & AF in tumor, \% \\
\hline BCOR & c.2751_2752del CCinsTT (p.Q918X) & 71.4 & 68.5 \\
KRAS & c.479T>C (p.V160A) & 47.3 & 52.6 \\
CDKN1B & c.400A $>$ G (p.K134E) & 25.1 & 25.9 \\
XPO1 & c.1711G $>$ A (p.E571K) & 23.5 & ND \\
DOT1L & c.481G $>$ A (p.D161N) & 6.6 & ND \\
DOT1L & c. $413 A>G(p . E 138 G)$ & 3.5 & ND \\
KRAS & c.34G $>$ C (p.G12R) & 0.8 & ND \\
KRAS & c.35G $>$ A (p.G12D) & 0.5 & ND \\
\hline
\end{tabular}

AF, allele frequency; ND, not detected.

Table 2 Summary of NK/T-cell lymphoma cases involving the penis since 2006

\begin{tabular}{lccccc}
\hline Refs, year & Age & Presentation & Treatment & Outcome & OS or PFS \\
\hline Ref (5), 2017 & 58 & Mass & CT & DOD & OS: 4 months \\
Ref (7), 2018 & 39 & Mass & Mass & CT & NR \\
Ref (6), 2008 & 48 & CT \& & NRonths \\
Ref (8), 2006 & 46 & Nodule, ulcer & Surgery \& CT & NR & OS: 1 year \\
\hline
\end{tabular}

OS, overall survival; PFS, progression-free survival; CT, chemotherapy; RT, radiotherapy; DOD, died of disease; CR, complete remission; PR, partial remission; AWR, alive with relapse; AWD, alive with disease; NA, not available; NR, not reported.

response rate (ORR) $67-77 \%$ and CR rate $50-66 \%$ $(10,11)$. The significance of local radiotherapy is not clear and whether testicular radiotherapy should be covered is debatable, which needs to be confirmed with further clinical studies. Although the exact value of autologous HSCT remains uncertain, numerous retrospective studies have shown that patients with advanced disease or high risk to recurrence can benefit from auto-transplantation, while allogeneic transplantation is currently in the exploratory stage because of its high treatment-related risks, and it's possible to try to treat refractory patients after autologous transplantation $(3,12)$. Chidamide, an innovative new drug independently developed in China, is designed to selectively inhibit the activity of HDAC1, 2, 3, and 10 following oral administration, and was approved by China Food and Drug Administration (CFDA) in 2014 to use in the secondline treatment of PTCL. Chidamide was tested to arrest cell cycle progression at the G0/G1 phase, and induced apoptosis in the NKTCL cell lines, which also suppressed the phosphorylation levels of proteins in the AKT/mTOR 
and MAPK signaling pathways and activated the DDR cell cycle checkpoint pathway, that is, the ATM-Chk2-p53-p21 pathway in vitro. Shi et al. analyzed the efficiency in $\mathrm{R} / \mathrm{R}$ ENKTL is about $19 \%(3 / 16)(13)$. We look forward more experiments to verify the therapeutic advantage of HDAC inhibitor in ENKTLs and such a good response to our patient may be a one-sided confirmation. To some degree, we suppose HDAC inhibitor as maintenance therapy is of value in keeping a long PFS in metastasis ENKTL patients who are not candidates for HSCT.

Exploring effective targets in the R/R ENKTLs is urgent and epigenetic dysregulation may broaden our understanding of ENKTL. Studies have shown that HDAC inhibitors are effective in B-cell lymphoma with mutations in EP300, CREBBP, EZH2 and MEF2B genes. To highlight in this patient is that $B C O R$ gene mutations occur specifically in ENKTL; retrospective statistical analysis of multiple genes shows a $B C O R$ mutation frequency of $32 \%$ (14). The patient's sample showed the BCOR gene p.Q918X exon 4 truncation mutation, which causes the stop codon encoded in advance to generate a truncated protein. The encoded product of the BCOR gene is a POZzinc finger structure-specific interaction transcription corepressor, which can be regulated by a target gene such as the proto-oncogene BCL-6 promoter sequence. Due to the high frequency and distortion patterns, as a tumor suppressor gene, BCOR may play an important role in the pathogenesis of ENKTL. Another gene CDKN1B, encodes p27 protein, which is a negative regulator of cell cycle, blocking cells from G1 to $\mathrm{S}$ phase; its low expression is a good prognostic marker of acute myelocytic leukemia (AML) but leads to a significant reduction in the survival rate of lymphoma and a triple increase in the risk of death (15).

Taken together, this case broadened the spectrum of differential diagnosis of penile tumors and exhibited the value of detailed history taking and comprehensive workup. The efficacy of HDAC inhibitor in ENKTL needs more clinical trials to confirm, but our case presented as long PFS as 12 months to prove its efficacy. Also, with the development of second-generation sequencing technology, gene detection is becoming increasingly important in guiding the therapy in relapsed/refractory NKTCL. This case may illuminate future treatment of similar cases or be part of value to further clinical trials.

\section{Acknowledgments}

Funding: None.

\section{Footnote}

Conflicts of Interest: All authors have completed the ICMJE uniform disclosure form (available at http://dx.doi. org/10.21037/apm-19-430b). The authors have no conflicts of interest to declare.

Etbical Statement: The authors are accountable for all aspects of the work in ensuring that questions related to the accuracy or integrity of any part of the work are appropriately investigated and resolved. All procedures performed in studies involving human participants were in accordance with the ethical standards of the institutional and/or national research committee(s) and with the Helsinki Declaration (as revised in 2013).Written informed consent was obtained from the patients for publication of this case report and any accompanying images.

Open Access Statement: This is an Open Access article distributed in accordance with the Creative Commons Attribution-NonCommercial-NoDerivs 4.0 International License (CC BY-NC-ND 4.0), which permits the noncommercial replication and distribution of the article with the strict proviso that no changes or edits are made and the original work is properly cited (including links to both the formal publication through the relevant DOI and the license). See: https://creativecommons.org/licenses/by-nc-nd/4.0/.

\section{References}

1. Haverkos BM, Pan Z, Gru AA, et al. Extranodal NK/T cell lymphoma, nasal type (ENKTL-NT): an update on epidemiology, clinical presentation, and natural history in North American and European Cases. Curr Hematol Malig Rep 2016;11:514-27.

2. Sun J, Yang Q, Lu Z, et al. Distribution of lymphoid neoplasms in China: analysis of 4,638 cases according to the World Health Organization classification. Am J Clin Pathol 2012;138:429-34.

3. Yong $\mathrm{W}$, Zheng $\mathrm{W}, \mathrm{Zhu} \mathrm{J}$, et al. L-asparaginase in the treatment of refractory and relapsed extranodal NK/T-cell lymphoma, nasal type. Ann Hematol 2009;88:647-52.

4. Wang X, Gong Z, Li SX, et al. Extranodal nasal-type natural killer/T-cell lymphoma with penile involvement: a case report and review of the literature. BMC Urol 2017;17:77.

5. Lan SK, Lin CW, Ho HC, et al. Penile metastasis secondary to nasal NK/T-cell lymphoma. Urology 
2008;72:1014-5.

6. $\mathrm{Li} \mathrm{Y,} \mathrm{Fu} \mathrm{X,} \mathrm{Wu} \mathrm{J,} \mathrm{et} \mathrm{al.} \mathrm{Penile} \mathrm{metastasis} \mathrm{secondary} \mathrm{to}$ nasal-type extranodal natural killer/T-cell lymphoma: a case report and review of the literature. Oncol Lett 2018;15:8034-8.

7. Yao HJ, Ying J, Wang Z, et al. One case report of primary penile malignant lymphoma (with a review of 24 case reports). Zhonghua Nan Ke Xue 2006;12:520-7.

8. Baldur-Felskov B, Hannibal CG, Munk C, et al. Increased incidence of penile cancer and high-grade penile intraepithelial neoplasia in Denmark 1978-2008: a nationwide population-based study. Cancer Causes Control 2012;23:273-80.

9. Barnholtz-Sloan JS, Maldonado JL, Pow-sang J, et al. Incidence trends in primary malignant penile cancer. Urol Oncol 2007;25:361-7.

10. Kwong YL, Kim WS, Lim ST, et al. SMILE for natural killer/T-cell lymphoma: analysis of safety and efficacy from the Asia Lymphoma Study Group. Blood 2012;120:2973-80.

Cite this article as: Fu C, Yang C, Li Q, Wang L, Zou L. Glans metastatic extra-nodal natural killer/T-cell lymphoma, nasal-type with HDAC inhibitor as maintenance therapy: a rare case report with literature review. Ann Palliat Med 2020;9(5):36023608. doi: 10.21037/apm-19-430b
11. Yamaguchi M, Kwong YL, Kim WS, et al. Phase II study of SMILE chemotherapy for newly diagnosed stage IV, relapsed, or refractory extranodal natural killer $(\mathrm{NK}) / \mathrm{T}$-cell lymphoma, nasal type: the NK-Cell Tumor Study Group study. J Clin Oncol 2011;29:4410-6.

12. Kwong YL. High-dose chemotherapy and hematopoietic SCT in the management of natural killer-cell malignancies. Bone Marrow Transplant 2009;44:709-14.

13. Shi Y, Dong M, Hong X, et al. Results from a multicenter, open-label, pivotal phase II study of chidamide in relapsed or refractory peripheral T-cell lymphoma. Ann Oncol 2015;26:1766-71.

14. Dobashi A, Tsuyama N, Asaka R, et al. Frequent BCOR aberrations in extranodal NK/T-cell lymphoma, nasal type. Genes Chromosomes Cancer 2016;55:460-71.

15. Erlanson M, Portin C, Linderholm B, et al. Expression of cyclin $\mathrm{E}$ and the cyclin-dependent kinase inhibitor p27 in malignant lymphomas-prognostic implications. Blood 1998;92:770-7. 